Résumés des conférences et travaux

\title{
Philologie et historiographie du Caucase chretien
}

Jean-Pierre Mahé

\section{(2) OpenEdition \\ Journals}

\section{Édition électronique}

URL : https://journals.openedition.org/ashp/3496

DOI : $10.4000 /$ ashp.3496

ISSN : 1969-6310

\section{Éditeur}

Publications de l'École Pratique des Hautes Études

\section{Édition imprimée}

Date de publication : 1 septembre 2020

Pagination : 44-53

ISSN : 0766-0677

\section{Référence électronique}

Jean-Pierre Mahé, "Philologie et historiographie du Caucase chretien », Annuaire de l'École pratique des hautes études (EPHE), Section des sciences historiques et philologiques [En ligne], 151 | 2020, mis en ligne le 09 juillet 2020, consulté le 06 juillet 2021. URL : http://journals.openedition.org/ashp/3496 ; DOI : https://doi.org/10.4000/ashp.3496 


\title{
PHILOLOGIE ET HISTORIOGRAPHIE DU CAUCASE CHRETIEN
}

\author{
Directeur d'études : M. Jean-Pierre MAHÉ, \\ membre de l'Institut
}

Programme de l'année 2018-2019: I. Les Saints Traducteurs arméniens : émergence d'un mythe. - II. Les juifs de Mcxeta dans la Conversion de la Géorgie : fiction romanesque et réalité historique.

I. Les premiers mots que recopièrent les apprentis traducteurs qui entouraient Maštoc ' en 405, juste après la mise au point définitive de l'alphabet arménien, furent l'incipit des Proverbes de Salomon : "Connaître la sagesse et le conseil, comprendre les paroles de la réflexion ». Rétrospectivement, ce qui n'était que le titre développé d'une gnomologie a pris, au cours des âges, le sens d'un vaste projet scientifique, le programme d'ensemble des traductions arméniennes, bibliques, patristiques et savantes.

La réalisation d'une tâche aussi vaste ne pouvait être l'œuvre d'une ou deux générations, mais de plusieurs siècles : le $\mathrm{v}^{\mathrm{e}}$ siècle pour la Bible et le début des traductions patristiques, les $\mathrm{VI}^{\mathrm{e}}-\mathrm{VII}^{\mathrm{e}}$ siècles pour les arts libéraux et certaines traductions patristiques. Après une interruption due à l'expansion musulmane et aux ruines $\mathrm{du} \mathrm{VIII}^{\mathrm{e}}$ siècle, le mouvement reprend du $\mathrm{X}^{\mathrm{e}}$ au $\mathrm{XV}^{\mathrm{e}}$ siècle jusqu'aux invasions de Tamerlan, embrassant la médecine, les arts appliqués et la théologie scolastique.

Néanmoins, dans la mémoire collective, l'acquisition de cette somme impressionnante de savoirs a été imputée en général aux Saints Traducteurs regroupés autour de Sahak et de Mesrop, dont on célébrait annuellement la fête. Comme le remarque Vardan, «beaucoup se mirent en route à leur suite, dans tous les siècles et toutes les époques ». Peu importe, en somme, de quand datent les diverses traductions, puisque leurs auteurs s'intègrent tous à un mythe intemporel, récit métaphorique de la construction de la science.

Vers le dernier tiers du $\mathrm{VI}^{\mathrm{e}}$ siècle, quand les Arméniens eurent traduit dans leur langue la Grammaire de Denys de Thrace et le Livre des Êtres, c'est-à-dire l'Organon d'Aristote, accompagné de savants commentaires des philosophes de l'Antiquité tardive, les écrits disponibles en arménien se répartirent d'eux-mêmes en deux groupes : « intérieurs » (nerkink') à la foi - c'est-à-dire bibliques et patristiques - et « extérieurs » (artakink'), c'est-à-dire profanes. Ces derniers ne se lisaient pas sans péril, mais ils semblaient indispensables à l'apprentissage des arts libéraux, base de

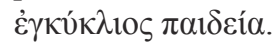

Pour limiter les risques, on pouvait prendre diverses précautions. En faire des « livres étroits » (nurb greank'), c'est-à-dire d'accès restreint, réservés à des élèves avancés, ou encore faire expliquer les matières profanes par des maîtres de "l'intérieur ». Néanmoins, même avec de telles garanties, il ne pouvait y avoir d'éducation complète sans apprentissage de «l'extérieur». 
C'est cette nécessaire sortie pédagogique que met en scène le récit du périple des Saints Traducteurs, relaté par Vardan vers 1257. «Envoyés chez les peuples de cantons étrangers de race et de langue (...) ils traversaient les mers et, volant d'une aile légère, ils montaient sur ces rapides coursiers de bois, saisissant aux brides leurs amples voilures, agitant leurs lanières déployées dans l'air ». L'idée de cette navigation remonte sans doute aux confidences de Movsēs Xorenac'i. Prétendant raconter ses propres aventures, Movsēs s'inspirait en fait du voyage d'étude, réel mais beaucoup plus limité, qu'Anania Širakac'i avait évoqué, en 660, dans la préface de son recueil de problèmes mathématiques. Alors que le savant, quittant seul sa patrie, s'était arrêté à Trébizonde, le chroniqueur, s'incluant lui-même dans un groupe de disciples de Sahak et de Mesrop, élargit leurs pérégrinations à l'ensemble du bassin méditerranéen. La figure du traducteur itinérant s'est également nourrie des colophons dont Step'anos Siwnec'i (vers 685-735) a muni les versions savantes qu'il a effectuées à Constantinople entre 712 et 718 .

En marge de ces données historiques, se sont échafaudées diverses fictions romanesques complétant le récit de Movsēs Xorenac'i. Par exemple, au $\mathrm{X}^{\mathrm{e}}$ siècle, les "Questions des saints docteurs d'Arménie, Movsēs et Dawit", adressées aux dyophysites ", racontent les aventures imaginaires des disciples de Sahak et de Mesrop envoyés à Athènes, appelés à construire la forteresse de Théodosiopolis et revenant à Constantinople pour disserter victorieusement contre le concile de Chalcédoine !

Citons encore un opuscule de date incertaine qui présente plusieurs points de contact avec le Panégyrique de Vardan.

Paroles du philosophe Dawit‘, qui connait parfaitement la philosophie théorique et pratique avec ses douze subdivisions. Originaire du canton de Hark', du village de Heret', il fut le disciple de Sahak et de Mesrop. On dit qu'il était le fils de la sœur de Movsēs, le père des rhéteurs, et l'un des soixante, choisis pour leur intelligence, leur belle voix et leur endurance, qui, cent onze ans après saint Grégoire, furent désignés pour découvrir l'écriture de cette nation et partirent à l'étranger pour étudier la philosophie et la rhétorique, ainsi que pour faire des traductions, à Alexandrie, à Athènes et dans d'autres provinces. Voici ceux qui partirent avec lui : Mambrē, le frère de Movsēs, Mušē et Ełišē, Arjan Arcruni et Ałan. Or, quand ils revinrent, beaucoup de temps était passé, beaucoup d'événements étaient survenus dans ce pays : saint Sahak avait été écarté de son siège patriarcal, Artašir avait été tué par le prêtre Surmak à cause de la trahison des princes, alors qu'on avait établi un prélat d'origine syrienne (...).

Ce banal résumé hagiographique, mélangeant à de maigres réminiscences historiques une tradition largement légendaire, montre que la réflexion de Vardan, dans son Panégyrique, ne s'oriente pas vers la reconstitution scrupuleuse des événements passés, mais vise surtout à valider un mythe de la mémoire collective, en exaltant les efforts et les sacrifices de ceux qui ont doté l'Arménie des savoirs qui l'ont hissée au plus haut niveau de la culture, sans rien devoir à l'arrogance des Grecs.

À la différence des séjours d'étude, rapportés par Koriwn, d'Eznik Kołbac'i et de Yovsēp" Pałnac'i à Édesse, et de Łewond et Koriwn à Constantinople, les pérégrinations prêtées au groupe des soixante Saints Traducteurs sont purement métaphoriques. Puisqu'ils ont étudié des sages " extérieurs ", la tradition a laissé croire qu'ils les ont rencontrés à Alexandrie, Byzance, Athènes ou ailleurs, dans toutes les 
métropoles du savoir. C'est pourquoi, grâce à eux, tout en demeurant dans la patrie, les moines qui lisent, commentent ou copient la version arménienne des écrits « extérieurs » font métaphoriquement le même voyage.

Leur parcours inclut : « la philosophie pratique et théorique, dont les parties sont au nombre de douze $»$. Typiques du programme des universités médiévales floris-

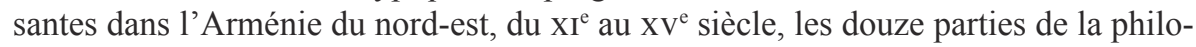
sophie se définissent ainsi : physique (1), mathématiques (2), théologie (3), éthique (4), économie (5), politique (6), arithmétique (7), musique (8), géométrie (9), astronomie (10), rhétorique (11) et les sept livres des philosophes païens (Grammaire, Isagogê, Définitions, Catégories, de Interpretatione, de Mundo, de Virtutibus).

Réparti sur sept ou huit ans, ce cursus studiorum se pratique au XIII ${ }^{\mathrm{e}}$ siècle, à l'université de Glajor, fondée vers 1282 par Nersēs Tarawnec'i, qui succède à Vardan Arewelc'i, en 1267, dans l'école de Xor Virap. Mais l'école monastique de Hałbat, où le Panégyrique des Saints Traducteurs est prononcé vers 1257, doit avoir aussi le même programme.

Suivant la formule de l'époque, le but des études est de parer les religieux d'une triple formation : " théorique, pratique et gnostique ». Classées en ordre de progression croissante, ces trois orientations de la pensée définissent une voie spirituelle de perfection monastique. Pour bien agir, il faut d'abord savoir ce que l'on fait, mais la pratique est évidemment supérieure à la théorie. Nous apprenons ainsi, dès le début du Panégyrique, que saint Sahak, «non seulement enseignait par la parole, mais accomplissait dans ses actes ce qu'il avait dit ».

Cependant les actions et la pratique en général relèvent de la conduite (vark'), observable de l'extérieur, et non pas des dispositions morales (bark') de "l'homme intérieur » qui, comme l'enseigne l'Apôtre, a des relations conflictuelles avec la chair dont il est revêtu. Existerait-il une voie qui permettrait de réconcilier l'intérieur et l'extérieur pour assurer le maintien durable d'une conduite vertueuse? C'est précisément ce que recherchent les gnostiques, au sens où l'entendait Évagre le Pontique.

Cela exige l'acquisition du discernement spirituel, c'est-à-dire d'un regard apaisé sur les tumultes, les meurtrissures, les catastrophes et les tragédies de l'univers. On ne saurait faire la paix en soi-même sans dépasser l'apparente contradiction entre ce cortège de maux et la foi en un Dieu bon et tout puissant. Le seul moyen d'y parvenir est de prendre de la hauteur, de replacer l'angoisse du moment présent dans une vue globale du monde et de la création.

Une telle sérénité ne s'acquiert que par étapes au prix d'un effort assidu. Avant d'y parvenir, Vardan a soin de laisser une large place à l'émotion, au deuil et à la cruelle déconvenue qui attendaient les Traducteurs rentrés en Arménie après la mort de leurs deux maîtres et le déclin de leur patrie, aux souffrances innombrables endurées par la nation depuis plus de huit siècles. Comment l'auditoire pourrait-il s'associer à la joie de la fête sans avoir d'abord déploré ces malheurs?

Mais la plainte est stérile si elle ne conduit pas à la pénitence que les Saints Traducteurs surent prolonger par une héroïque ascèse pleine de promesses pour le bien de l'Église et du monde. La vision dont bénéficia saint Sahak, sa mort édifiante, ainsi que la croix lumineuse qui accompagna Mesrop jusqu'à la tombe confirment que les promesses divines seront tenues, que la présence invisible de la Providence se dévoile à qui sait la contempler. 
Cet exercice de contemplation " gnostique » nous est offert dans l'éloge de Sahak et de Mesrop, inventeurs de l'écriture. Il suffit d'ouvrir les yeux pour constater qu'ils sont présents dans l'assemblée, à travers l'innombrable progéniture spirituelle que leur a donnée le Seigneur. Mais surtout, les lettres elles-mêmes qui ont le pouvoir de manifester les pensées invisibles, de fixer les paroles qui passent, contiennent un mystère divin, que l'on peut entrevoir à travers un récit à mi-chemin entre le mythe et l'histoire.

Tentons de développer les ellipses de ce mythe. L'écriture est avant tout un remède contre l'oubli, conséquence funeste du péché originel et de l'expulsion du Paradis. C'est ainsi que les nations païennes, descendance des Caïnites, oublièrent Dieu, se forgèrent des idoles, adorant la matière inerte et l'œuvre de leurs mains. Mais la vraie progéniture d'Adam, c'est-à-dire les fils de Seth, firent du mieux qu'ils purent pour conserver pieusement « les grâces et les sages pensées que Dieu avait accordées au premier homme ». Néanmoins, ils étaient, eux aussi, d'autant plus menacés par l'oubli que l'abrègement de la vie humaine ordonnée par Dieu eut pour conséquence d'entraver la transmission de la mémoire entre les générations.

C'est pourquoi «Énoch, qui était le $7^{\mathrm{e}}$ après Adam, et fut, par un prodigieux miracle, ravi dans la gloire ", inventa la première écriture "comme moyen pour ranimer dans notre nature le souvenir des prodiges divins ». Malheureusement, elle se perdit pendant le Déluge et dut être réinventée au temps de la Tour de Babel sans doute pour faciliter les travaux des ingénieurs. Les Chaldéens s'en emparèrent et la notèrent sur des tablettes d'argile arrachées à la terre encore toute humide du Déluge. Diverses nations païennes les imitèrent, et, par l'usage profane qu'elles en firent, dévoyèrent complètement la pieuse invention d'Énoch. Heureusement les Hébreux, détachés des impies par la migration d'Abraham, reçurent de Dieu, par «le grand Moïse, premier Prophète », des lettres sacrées destinées à écrire la Loi.

L'Incarnation du Verbe ouvre un âge nouveau qui restaure l'ancien. Familiers du Fils de Dieu, les Apôtres conservent de lui, après l'Ascension, une mémoire aussi vive que celle qu'Adam gardait du Paradis. Mais, comme la fréquentation de leur maître les avait lavés de leurs vices, c'est-à-dire délivrés de la tache originelle, leur mémoire était devenue ineffaçable. Aussi « ne se pressèrent-ils pas de transmettre aux hommes par écrit les prodiges de l'Économie du Verbe de Dieu, car ils désiraient que tous devinssent comme eux, conservant sans oubli les souvenirs dans leur esprit ». Mais la réalité les rattrapa et les contraignit à changer d'avis en usant de l'écriture la plus courante, c'est-à-dire l'alphabet grec emprunté aux païens.

Grâce aux Saints Traducteurs les Arméniens ont eu la chance d'échapper à cette périlleuse compromission. Car leur alphabet leur fut donné directement d'en haut, tout comme celui de Moïse, en sorte qu'ils furent « affranchis des ténèbres de l'ignorance » et virent « la lumière de la véritable connaissance de Dieu ».

Il convient ici de rappeler l'interprétation théophanique des lettres arméniennes, qui s'est affirmée dès l'origine dans l'historiographie. Certes Movsēs Xorenac'i innove en prêtant à Mesrop une vision de la dextre divine écrivant des caractères qu'il n'a plus qu'à recopier. Mais d'une façon plus subtile Koriwn suggérait avant lui une inspiration trinitaire de l'alphabet: de ce fait, Maštoc' devenait égal ou même supérieur à Moïse. Comme lui, il avait rencontré Dieu, qui lui avait fait don de l'écriture de sa nation. 
Par contraste, les deux autres alphabets attribués à Maštoc', le géorgien et l'albanien, sont présentés non comme des dons divins mais comme des inventions humaines, fruits de l'expérience personnelle de leur inventeur. Ce sont en quelque sorte les restes de la grâce. Comme le dit Vardan, sous forme de proverbe : « de la chasse du lion, maint renard se repaît ». Pourquoi cette discrimination entre les Arméniens et les autres peuples?

La fierté nationale ne s'embarrasse guère de justification. Remarquons pourtant que la version $B$ du Panégyrique de Vardan suggère un début d'argumentation en liant l'origine de l'écriture aux huit âges du monde.

$1^{\text {er }}$ âge : écriture d'Énoch; $2^{\mathrm{e}}$ âge : écriture de la Tour de Babel; $3^{\mathrm{e}}$ âge : tablettes des Chaldéens; $4^{\mathrm{e}}$ âge : alphabet des Latins; $5^{\mathrm{e}}$ âge : Moïse et les Hébreux; $6^{\mathrm{e}}$ âge : Sahak et Mesrop; $7^{\mathrm{e}}$ âge : écriture commune à tous; $8^{\mathrm{e}}$ âge $:$ béatitude éternelle.

Pour fruste qu'il soit, ce schéma chronologique rejoint le mythe de la grande semaine cosmique. De même que Dieu créa le monde en six jours, puis se reposa le septième, ainsi le monde créé a connu six âges de mille ans, comme chacun des jours de Dieu. Au septième âge, le Christ régnera sur terre parmi les justes, et le huitième s'ouvrira sur la béatitude céleste.

Remède à l'oubli, l'écriture n'apparaît pas avant la fin du premier âge, juste à la veille du Déluge. De même, c'est à la fin du septième âge « et à l'entrée du huitième » que « l'art merveilleux d'écrire la parole deviendra commun à tous et n'aura plus besoin d'être appris ». Le huitième âge marque normalement un retour aux origines. Dans l'Ancien Testament c'est l'époque de Noé, fondateur d'une humanité nouvelle après le Déluge.

Dans le Nouveau Testament, le huitième âge est forcément lié au huitième jour, c'est-à-dire à la résurrection du Christ, qui a lieu après le sabbat, au début d'une nouvelle semaine. Du point de vue eschatologique, l'événement coïncide avec la restauration de l'immortalité primordiale. De ce fait, les élus deviendront comme Adam avant la chute : dénués d'oubli et de sommeil, jouissant continuellement de la présence ou de la mémoire de Dieu. L'écriture de la fin du septième âge, qui s'inscrira d'elle-même dans l'esprit de tous, symbolise l'abolition de l'oubli.

Malgré l'extrême concision du récit de $B$, on croit comprendre que, selon le mythe, l'écriture arménienne, inventée au sixième âge, bien après celle des autres peuples (Chaldéens, Latins, mais aussi bien Grecs et Syriens), remplit une fonction analogue. C'est un don divin qui survient juste à temps pour empêcher l'oubli des vraies traditions apostoliques, menacées, du point de vue des Arméniens, par les hérésies christologiques de Nestorius, d'Eutychès et du concile de Chalcédoine, qui ne cessent d'être débattues de 451 à 726, lorsque Yovhannēs Awjnec'i fixe la doctrine de l'Église arménienne.

En 553, quand les miaphysites syriens du Tur 'Abdin sollicitent auprès du catholicos Nersēs II la consécration épiscopale d'Abdišō, ils persuadent leurs interlocuteurs que, depuis le concile de Chalcédoine, toutes les Églises, sauf les Arméniens et leur propre communauté, ont versé dans l'hérésie de Nestorius, en sorte qu'Arméniens et Syriens miaphysites sont les derniers chrétiens authentiques. L'historiographie arménienne de l'expansion arabe en Palestine impute pareillement aux déviations christologiques la défaite byzantine du Yarmouk en 636. 
De même que, selon les historiens arméniens des $\mathrm{VII}^{\mathrm{e}}-\mathrm{VIII}{ }^{\mathrm{e}}$ siècles, les Hébreux ont perdu leur Ville Sainte et leur sol ancestral pour avoir crucifié le Christ, les Grecs et les autres chrétiens - jusqu'alors nouvel Israël, héritier de l'ancien - ont dû céder cet héritage aux fils d'Ismaël, en châtiment d'un crime équivalent : au concile de Chalcédoine, ils ont « coupé le Christ en deux », séparant sa nature humaine de sa nature divine.

Naturellement la Providence avait depuis toujours la prescience de cette trahison. C'est pourquoi, à la veille des funestes controverses qui devaient consommer ce forfait, elle avait fait don aux Arméniens d'une écriture d'inspiration divine. Ainsi, comme les Hébreux avaient jadis, grâce à l'écriture de Moïse, préservé la connaissance du vrai Dieu au milieu des idolâtres, les Arméniens, munis de l'alphabet révélé à Mesrop Maštoc ', le nouveau Moïse supérieur à l'ancien, avaient maintenu, seuls au monde, la doctrine authentique de l'ineffable union de Dieu et de l'humanité, dans «l'unique nature du Verbe incarné ».

II. L'ensemble des textes réunis au Moyen Âge sous le titre Conversion de la Géorgie, nous est transmis par trois manuscrits : S̆at'berd ( $\mathrm{x}^{\mathrm{e}}$ siècle), découvert en 1888; C̆'eliš ( $\mathrm{XII}^{\mathrm{e}}$ siècle), découvert en 1903; NSin 50 ( $\mathrm{IX}^{\mathrm{e}}-\mathrm{X}^{\mathrm{e}}$ siècle), découvert en 1996 (à quoi s'ajoutent quelques fragments dans NSin 48). Chacun de ces trois codices représente une rédaction différente. Pour l'essentiel, le dossier se compose de deux unités narratives : tout d'abord un récit continu, que nous proposons d'appeler "Chronique des trois empereurs » (Alexandre, Constantin et Héraclius), achevé en 630 ; puis six témoignages croisés rédigés à Jérusalem vers la fin $\mathrm{du} \mathrm{IX}^{\mathrm{e}}$ siècle et fictivement attribués à Salomé d'Užarma, belle-fille de Mirian, le premier roi chrétien, l'évangélisatrice Nino, racontant elle-même sa vie, puis la pieuse juive Sidonie, son père Abiatar, de classe sacerdotale, le prêtre chrétien Iak'ob et le roi Mirian.

Trois femmes sont suivies de trois hommes, et les deux groupes sont disposés dans un ordre rigoureusement symétrique : païenne, chrétienne, juive, puis juif, chrétien, païen. Cette composition en abîme (on pourrait presque dire cette " gammatria ») dirige l'attention sur les deux personnages centraux, Sidonie et Abiatar, juifs convertis au christianisme, qui éclairent par leurs propos aussi bien que par leur personne, la signification cachée du baptême des Ibères, à quoi ils ont assisté.

La place éminente que l'auteur chrétien leur accorde, à eux-mêmes et à leur ancêtre, le prêtre Elioz, contemporain du Christ, étonne d'autant plus qu'elle contraste avec les orientations plutôt anti-judaïques de l'évangile. Au contraire notre chroniqueur s'efforce d'établir que les juifs de Mcxeta - mais aussi bien tout le peuple juif, au-delà de ses dirigeants - n'ont aucune responsabilité dans la mort du Sauveur. Donnant la parole à Sidonie, fille d'Abiatar, qui représente, en tant que prêtre, toute la lignée d'Israël, depuis Noé jusqu'à Abraham, Moïse et Josué, homonyme de Jésus, il lui fait raconter l'histoire de son ancêtre Elioz, témoin de la Passion :

Je vais vous dire un récit, que je connais à la fois par la lecture des livres et par le témoignage de mon père. Au temps où régnait Hérode, nous entendîmes ici la nouvelle que les Perses avaient pris Jérusalem (...).

Cependant, quelque temps après arriva de Jérusalem une autre proclamation, mais rassurante, adressée à tous les pays : «les Perses ne sont pas venus en conquérants de 
la contrée, mais au lieu de cuirasses, d'armement et de moyens de transport (pour le butin), ils ont apporté de l'or jaune, de la myrrhe qui guérit promptement les blessures et du benjoin parfumé : voilà tout ce qu'avait chacun de leurs cavaliers. Les rois euxmêmes en tenaient chacun une charge et ils cherchaient un certain enfant nouveau-né, fils de David, qu'ils trouvèrent comme un pèlerin, fils d'une pèlerine, né en temps et en lieu inopportuns, comme il arrive souvent à ceux qui sont en déplacement. Ils vinrent auprès de cet enfant, se prosternèrent devant lui et lui offrirent tous leurs présents. Puis ils repassèrent la montagne et s'en allèrent en paix. Ne craignez rien, juifs : moi, Hérode, j'ai fait rechercher cet enfant, et je ne l'ai pas trouvé, ni lui, ni sa mère. Toutefois, à présent, je dirigerai le glaive contre tout garçon de deux ans et en dessous : il périra bien, lui aussi avec eux ».

Plus encore que du sobre récit de Matthieu sur la visite des mages à la crèche et le massacre des enfants de Bethléem, cette paraphrase pittoresque des thèmes évangéliques - véritable haggadah néo-testamentaire - rappelle certains apocryphes, comme la Caverne des Trésors, traduite en Géorgien au VIII ${ }^{\mathrm{e}}$ siècle, d'après une ancienne version karšuni.

Mais, dans notre dossier, la réécriture fantaisiste de la nativité est censée parvenir aux juifs de Géorgie, contemporains du Christ. Elle s'adresse à une colonie diasporique, dont on nous décrit la structure sociale et religieuse. D'après le témoignage d'Abiatar, les juifs de la ville royale, sans doute des commerçants, habitaient, comme son ancêtre Elioz, un quartier extérieur situé en bordure du Mt'k'uari (Kura), à l'ouest de l'enceinte, près de la porte des Mages, où était le sanctuaire d'Armazi (Ahura Mazda). Ils y avaient un petit lieu de culte et un cimetière. Un quartier juif existait aussi dans la ville d'Urbnisi, où Nino passe avant d'arriver à Mcxeta.

En dehors des familles sacerdotales, dont certaines se sont fixées à Bodi, où sera plus tard enterrée Nino, il y a aussi des scribes, qui ont une école à K'odis-C'q'aro, et des interprètes targumiques à Sob-K'anani. Ces derniers ne sont pas de simples traducteurs, qui expliqueraient en géorgien les textes hébraïques incompréhensibles à leurs coreligionnaires. En effet, les juifs de Géorgie parlent araméen. C'est en cette langue que Nino s'entretient avec eux, dès qu'elle arrive à Urbnisi. L'activité des interprètes consiste donc à étudier ou à élaborer, avec l'effort exégétique que cela suppose, des targums araméens de l'original hébreu.

Établie au premier siècle avant notre ère, cette colonie entretient de nombreux échanges avec la Palestine, s'informe régulièrement du sort de la ville sainte et correspond avec les autorités religieuses ou civiles. Bien qu'elle s'insère dans une trame narrative assez fantaisiste, l'idée d'une lamentation envoyée aux diverses communautés de la diaspora, sous forme de lettre circulaire, est, en elle-même, assez vraisemblable. À l'image de la lamentation attribuée à Jérémie, qui déplore la prise de Jérusalem en $\sim 587$, le judaïsme médiéval a produit de nombreuses complaintes sur les massacres et les tribulations qui ont endeuillé son histoire. Ici, malgré le contenu apocryphe du message, la description des destinataires présente une indéniable cohérence historique. On ne saurait douter de l'existence ancienne de la communauté juive de Mcxeta. Si l'auteur chrétien, qui cherche à la défendre, ne recourt pas aux textes canoniques, c'est parce qu'il se défie, à juste titre, de leur orientation anti-judaïque. Cependant, une réécriture subtile de l'évangile peut conduire à des conclusions totalement opposées. 
Rapportant la suite des propos de son père, Sidonie raconte que, trente ans plus tard,

le prêtre Anne écrivit de Jérusalem à Ozias, le père de mon père. Il disait : « Ce même enfant, près de qui les rois perses étaient arrivés avec des présents, est maintenant devenu adulte. Il a atteint l'âge de trente ans. Toutes les tribus d'Israël venaient dans l'eau du Jourdain auprès du fils de Zacharie, et Elioz, le frère de ta grand'mère maternelle, y fut également » (...). Trois ans plus tard, arriva de Jérusalem un ordre du roi Hérode (...) : «Voici maintenant que s'est levé un homme, nommé Jésus qui, de son propre chef, appelle Dieu son père, et prétend être lui-même comme Dieu. Venez tous assister à sa mort, afin que nous accomplissions le commandement de Dieu et de Moïse ».

Dans notre chronique, comme dans les miniatures des évangéliaires, la vie publique du Christ est réduite à deux images : le baptême, qui marqua le début de sa prédication, et la condamnation à mort, qui y mit fin. De nouveau, l'auteur s'éloigne délibérément des évangiles canoniques. Le seul signe que ceux-ci décrivent est la descente de l'Esprit sous forme d'une colombe. Dans les synoptiques, Jésus est seul bénéficiaire de cette vision, et il entend aussi la voix du Père. D'après Jean, le Baptiste aperçoit la colombe, mais il n'entend pas la voix. En contraste avec cette paisible évocation, notre texte suggère que le Christ, venu en dernier après toutes les tribus d'Israël, a suscité des prodiges effrayants, qui ont épouvanté le fils de Zacharie et que les grands prêtres ont soigneusement cachés au peuple pour étouffer leur évidente signification messianique. Par exemple, le Jourdain a coulé en sens contraire. La tradition apocryphe attribue à Jésus ce miracle opéré par son homonyme Josué, quand il fit traverser le fleuve à l'Arche d'alliance. Le Christ est porteur d'une alliance nouvelle, et sa venue dans le monde inverse l'ordre ancien. Le tremblement de terre qu'il provoque rappelle d'abord les prodiges survenus après la mort du crucifié : en effet, le baptême - descente dans le monde inférieur - laisse pressentir la descente aux enfers, mais l'ébranlement des montagnes et le frémissement des collines annoncent aussi bien la fin des temps, le salut eschatologique. Voilà le message que, selon l'auteur, les princes des juifs n'ont pas voulu entendre

Elioz, notre ancêtre, poursuit le narrateur, partit du Kartli avec tous les juifs. Tout ce que nous savons maintenant par les évangiles du Christ s'accomplit. Mais la tunique échut par le sort à ce pays-ci, et ce fut Elioz qui la rapporta. Entre-temps, quand sa mère entendit le bruit du bourreau qui frappait les clous sur la croix, à Jérusalem, avec un marteau de forgeron - car le bruit s'entendit jusqu'ici - cette femme poussa un cri déchirant et dit : «Adieu, royaume des juifs, car vous avez tué le Sauveur et le Rédempteur, et désormais vous vous êtes rendus ennemis du Créateur! Malheur à moi, qui ne suis pas morte assez tôt pour n'avoir pas entendu ce bruit, ou qui ne reste pas assez longtemps pour voir la lumière rayonner sur les païens, et la gloire du peuple d'Israël! » Sur ces mots, la mère d'Elioz mourut. Quant à Elioz, il rapporta, à Mcxeta, dans notre maison, la tunique du Sauveur Jésus Christ et il apprit que sa mère était morte en disant telle parole. Cependant sa sœur venait à sa rencontre, baignée de larmes amères, comme du sang. Elle se jeta au cou de son frère, lui prit la tunique et la serra contre sa poitrine. Aussitôt, elle rendit l'âme à cause de ces trois afflictions : la mort de sa mère, et surtout la douleur de la mort du Christ et le désir de sa tunique. Alors il y eut un grand trouble 
à Mcxeta entre les rois, les princes et tout le peuple. Voyant la tunique de Jésus, le roi Amazael voulait s'en saisir, mais craignant de toucher cette femme morte, il eut peur et n'osa pas. Elioz enterra sa sœur, tenant encore la tunique dans ses mains.

Dans la logique d'un récit entièrement gouverné par les symboles, le pieux Elioz, qui reçoit le baptême de Jean, est voué d'avance, quand il part pour Jérusalem, à hériter de la sainte tunique, cause ultime et instrument providentiel de la conversion des Ibères. En effet, il porte le même nom que le prophète Élie, dont il descend en ligne maternelle. Ce sont les ancêtres de sa mère qui ont rapporté à Mcxeta le manteau d'Élie et l'ont enterré en un lieu où s'élèvera plus tard l'autel principal de la Colonne vivante. Jésus enseigne que Jean-Baptiste est l'Élie qui doit venir. De même que l'ancien Élie lui rend témoignage lors de la Transfiguration, le nouvel Élie est son précurseur, envoyé pour préparer la voie. Bien plus, en préférant la veuve de Sarepta à toutes les veuves d'Israël, Élie annonce le salut des nations, qui sera réalisé par le Christ. Celui qui détient le manteau du prophète est symboliquement le plus qualifié pour recevoir le vêtement du crucifié et en faire bénéficier la lointaine Géorgie, sombre pays de K'edar, plongé dans les brumes du nord et les ténèbres de l'idolâtrie.

Le manteau d'Élie a encore un autre pouvoir. Il protège de toute contagion l'envoyé de la Providence, contraint d'emprunter le chemin des pécheurs et d'assister au conseil des méchants. En effet, en nous indiquant l'ascendance de la mère d'Elioz, l'auteur montre bien qu'elle a hérité du don prophétique de son ancêtre Élie. Non seulement elle rappelle le mystère de l'aveuglement des sages d'Israël et de l'illumination des Gentils, qui est l'essence même de la prophétie, comme l'affirme la Caverne des Trésors, mais elle entend jusqu'à Mcxeta les coups de marteau qui résonnent depuis le calvaire. Alors que Pilate, prévenu par un songe de son épouse, " de ne pas se mêler des affaires de ce juste », accepte néanmoins de le supplicier à la demande de la foule, Elioz, averti par l'intuition prophétique de sa mère, trouve moyen de se soumettre à la loi en déférant à la convocation du roi, tout en s'opposant au projet criminel d'Hérode. Il se désole de la mort du condamné au point de recueillir son vêtement au pied de la croix.

Par conséquent, si l'on prend soin de rassembler tous les indices que l'auteur a volontairement dispersés et cachés dans diverses remarques incidentes du texte, il devient évident qu'Elioz et les juifs de Mcxeta qui l'ont accompagné, ne sont en aucune façon responsables de la mort du Christ. Ils se sont opposés à sa condamnation et ils en ont souffert pour eux-mêmes et pour toute leur famille. La mère d'Elioz succombe aux coups des crucifieurs. Durement éprouvée, sa sœur, la grand'mère d'Abiatar, meurt d'émotion en recevant la sainte tunique. Comment imaginer que des juifs, dévoués à la loi de leurs pères, qui rapportent à Mcxeta une relique, devenue plus tard le motif surnaturel de la conversion des Ibères, puissent être, de près ou de loin, associés aux bourreaux du Christ? Assurément, cela est impossible. Mais qu'en est-il alors du grief de déicide, formulé au Moyen-âge par beaucoup de sociétés chrétiennes à l'égard des juifs dans leur ensemble? Faut-il comprendre que les juifs de Mcxeta en sont exempts par exception ou que ce grief est absolument sans fondement?

Les juifs mis en scène dans cette fiction apologétique chrétienne mentionnent bien le commandement apocryphe de Moïse de suspendre au bois le blasphémateur, mais ils ne lui accordent plus aucune valeur. Tout comme la mère d'Elioz l'avait fait au 
moment de la condamnation du Christ, ils distinguent entre le royaume des juifs et le peuple dans son ensemble. Comme juifs de la diaspora, établis à Rome, siège des oppresseurs, en Égypte, théâtre de l'exode et à Mcxeta, terre d'exil - ils sont bien placés pour savoir que le royaume ne se confond pas avec Israël. Les autorités temporelles et spirituelles ont failli : voilà pourquoi le royaume est anéanti et les prophètes se taisent. Mais la nation n'est pas dépossédée de son histoire. Elle garde la mémoire de ses erreurs et de ses repentirs passés. Elle conserve la faculté de revenir à Dieu par la pénitence.

Plutôt qu'une singularité du judaïsme géorgien, l'histoire d'Elioz expose un modèle différent de rapports entre les juifs et les chrétiens : au lieu de l'affrontement, la reconnaissance et le respect d'un commun héritage historique et spirituel. 